\title{
Impact of nasogastric tube feeding on swallowing function in patients with dysphagia: a pilot study
}

\author{
${ }^{1}$ Yu Sang Jung $M D,{ }^{2}$ Min Young Kim $M D P h D,{ }^{2}$ Kyunghoon Min $M D P h D,{ }^{2}$ Jong Moon Kim $M D$, \\ ${ }^{3}$ Eun Young Han $M D P h D,{ }^{4}$ Kye Hee Cho $M D P h D,{ }^{1}$ Sang Hee Im $M D P h D$ \\ ${ }^{1}$ Department and Research Institute of Rehabilitation Medicine, Severance Hospital, Yonsei University \\ College of Medicine, Seoul; ${ }^{2}$ Department of Rehabilitation Medicine, CHA Bundang Medical Center, \\ CHA University, Seongnam, ${ }^{3}$ Department of Rehabilitation Medicine, Jeju National University, Jeju, \\ Jeju Special Self Governing Province; ${ }^{4}$ Department of Rehabilitation Medicine, CHA Ilsan Hospital, \\ CHA University, Goyang, South Korea
}

\begin{abstract}
During dysphagia treatment, direct oral swallowing therapy is applied to some patients temporarily fed via nasogastric tube. However, the risk of aspiration in oral swallowing while nasogastric tube in situ may be disregarded in a standard videofluoroscopic swallowing study performed without a nasogastric tube. To evaluate the diagnostic significance of nasogastric tube in situ videofluoroscopic swallowing study of nectar and pureed diet compared to the standard videofluoroscopic swallowing study without nasogastric tube. Videofluoroscopic swallowing study records of dysphagia patients conducted between June and August 2017 in a university hospital were collected for review. Rosenbek's penetration-aspiration scale, diagnostic criteria of aspiration were used to define aspiration. videofluoroscopic dysphagia scale for videofluoroscopic swallowing study with or without nasogastric tube were compared for nectar and pureed diet swallowing. Patients had various duration of nasogastric tube feeding. Paired T-test comparing the videofluoroscopic dysphagia scales for videofluoroscopic swallowing study with or without nasogastric tube revealed significant aggravation of swallowing dysfunction in nectar drinking while nasogastric tube in situ. This aggravation was noted in 19\% $(n=4)$ of patients who suffered from stroke regardless of nasogastric tube duration. Nasogastric tube in situ videofluoroscopic swallowing study, at least of nectar drinking could be beneficial in selecting a safe candidate for direct oral swallowing therapy in conjunction with the conventional nasogastric tube removed videofluoroscopic swallowing study.
\end{abstract}

Keywords: Nasogastric tube, videofluoroscopic swallowing study, dysphagia, aspiration

\section{INTRODUCTION}

Oropharyngeal dysphagia is one of the common and life-threatening symptoms of neurological disorders. At least $50 \%$ of patients with ischemic and hemorrhagic stroke ${ }^{1}$ and $60 \%$ of those with traumatic brain injury ${ }^{2}$ experience dysphagia. It is also an important risk factor for pneumonia in patients with Parkinson disease, which is known as the most frequent cause of mortality. ${ }^{3}$ Patients with motor neuron disease, neuromuscular junction disease, and inflammatory muscle disorders also present with dysphagia. ${ }^{4,5}$ Other than specific diseases, aging itself is a well-known risk factor for dysphagia and is present in $33 \%$ of the population aged $>80$ years. ${ }^{6}$
Nasogastric tube (NGT) feeding is often imperative in patients with dysphagia to maintain adequate nutritional support. Many patients with dysphagia from various etiologies depend on tube feeding until deficits are resolved or swallowing function is improved. ${ }^{7}$ Early initiation of swallowing treatment is recommended, with reliable evidence, for regaining swallowing function even in patients temporarily fed via NGT. ${ }^{7}$ Indeed, many patients demonstrate improved swallowing function to return to oral feeding after receiving early swallowing therapy. ${ }^{8}$ Dysphagia therapies to improve swallowing function include direct and indirect interventions, wherein direct therapy involves direct oral swallowing of food

Address correspondence to: Kye Hee Cho MD, PhD, Department of Rehabilitation Medicine, CHA Ilsan Medical Center, CHA University, 1205, Jungang-ro, Ilsandong-gu, Goyang, Gyeonggi-do, 10414, Republic of Korea. Tel: +82-54-4509649, Email: jacquilline@gmail.com. Sang Hee Im, MD, PhD, Department and Research Institute of Rehabilitation Medicine, Severance Hospital, Yonsei University College of Medicine, 50-1 Yonsei-ro, Seodaemun-gu, Seoul, 03722, Republic of Korea. Tel: +82-2-22283721, E-mail: dongin32@ naver.com

Date of Submission: 26 August 2020; Date of Acceptance: 4 September 2021

https://doi.org/10.54029/2021smr 
material.

Previously, studies have encountered aspiration events during direct swallowing therapy in some patients fed with NGT who were considered candidates of direct therapy according to videofluoroscopic swallowing study (VFSS) without NGT. In the past, the impact of NGT on swallowing function has been investigated in a few studies that demonstrated negligible effects.9-11 However, these results cannot fully guarantee the safety of direct swallowing therapy with NGT in situ. Although aspiration pneumonia does not necessarily occur in every aspiration incidence, changes in swallowing function with or without NGT may indicate aspiration-prone conditions for direct swallowing therapy.

Therefore, as an attempt to ascertain the safety of direct swallowing with NGT in situ during dysphagia therapy, patients are assessed several times with or without NGT for pureed diet and nectar in VFSS in our institute. This retrospective case-control study on VFSS results aimed to determine whether NGT affected the swallowing function of patients with dysphagia.

\section{METHODS}

Results of VFSS conducted between June and August 2017 at a secondary university hospital were obtained for this study. Among 32 patients who underwent VFSS during the period, those without NGT in situ VFSS or no follow-up were excluded. The overall VFSS results evaluated with or without NGT were analyzed in 21 (men: $7,33.3 \%$ ) patients with dysphagia from various etiologies (Figure 1, Table 1). All these patients received dysphagia therapy by an experienced occupational therapist according to the VFSS results. The outcome of dysphagia therapy was also collected. The recorded images of VFSS was assessed by a physiatrist. The VFSS protocol of the study facility was based on Logemann's study. ${ }^{12}$ The original VFSS was performed by a single physiatrist in the fluoroscopic radiography room. Swallowing function was initially evaluated with NGT for pureed diet and nectar in a sitting position. Then, the conventional VFSS without NGT consisting of variable degrees of food consistency and texture including pureed diet and nectar were performed consecutively. The results of conventional VFSS excluding pureed diet and nectar swallowing are not included in this study. After VFSS, the lateral views of the oropharynx and larynx were obtained. The test was discontinued if aspiration was detected on the fluoroscopic image. Fluoroscopic images were stored on the server system, and the physiatrist re-evaluated the results based on the recorded images.

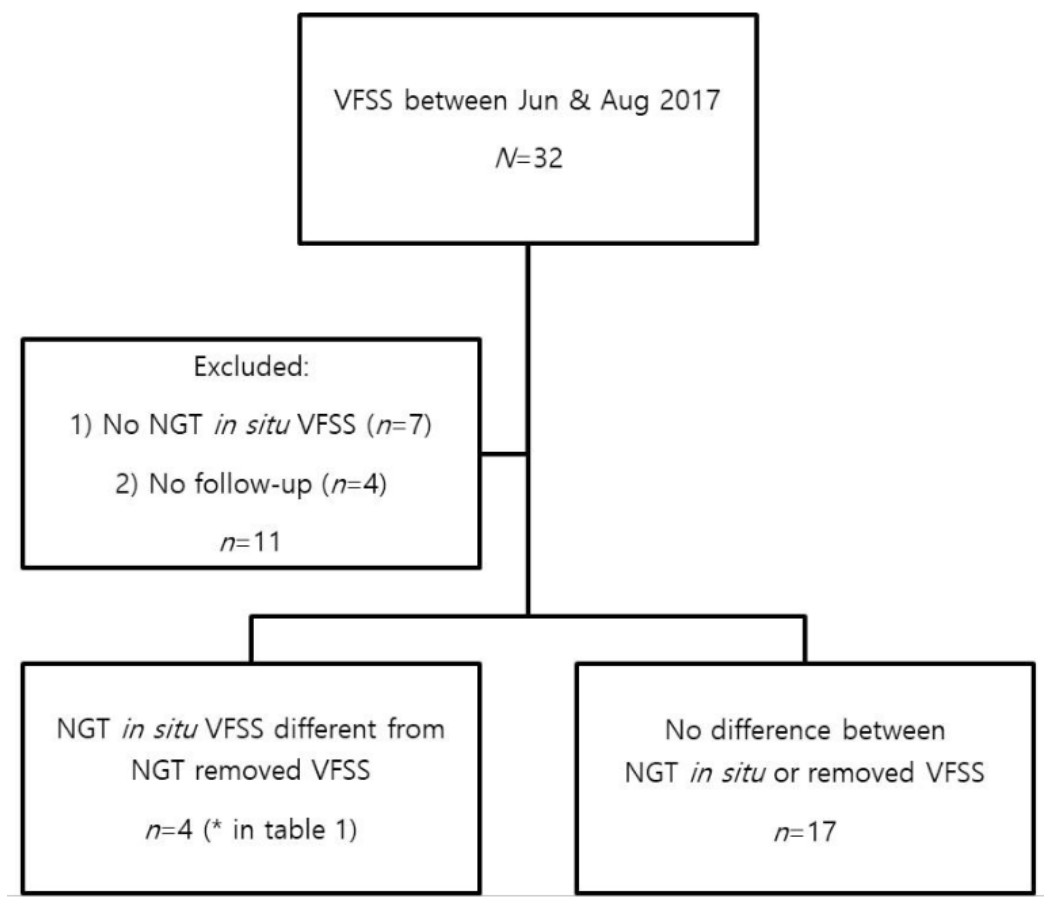

Figure 1. Flow chart showing the patient selection 


\begin{tabular}{rrrlccc}
\hline No. & Sex & Age & \multicolumn{1}{c}{ Etiology } & $\begin{array}{c}\text { Aspiration/ } \\
\text { silent } \\
\text { aspiration }\end{array}$ & $\begin{array}{c}\text { NGT } \\
\text { duration } \\
\text { (week) }\end{array}$ & PAS \\
\hline 1 & F & 59 & Lt thalamic ICH & $+/+$ & 12 & 8 \\
2 & M & 80 & Oropharyngeal cancer & $+/-$ & 3 & 6 \\
3 & F & 85 & Bilateral frontal infarct & $+/+$ & 20 & 8 \\
$* 4$ & F & 51 & Lt BG infarct & $+/+$ & 2 & 8 \\
5 & F & 75 & Rt parietal ICH & $+/+$ & 12 & 8 \\
6 & F & 90 & Bed ridden s/p Rt total hip replacement & $-/-$ & 1 & 2 \\
7 & F & 86 & Parkinson's disease & $+/-$ & 1 & 6 \\
8 & F & 56 & Guillain Barre Syndrome & $-/-$ & 8 & 2 \\
9 & M & 84 & Rt frontal infarct & $+/+$ & 6 & 8 \\
10 & F & 76 & Parkinson's disease & $+/+$ & 3 & 8 \\
11 & M & 65 & Lung Cancer metastasis s/p ChemoRTx & $+/-$ & 4 & 6 \\
$* 12$ & F & 88 & Lt thalamic ICH & $+/+$ & 12 & 8 \\
13 & M & 66 & Parkinson's disease & $+/+$ & 6 & 6 \\
$* 14$ & M & 84 & Old CVA (10, 4YA), asphyxia on RD & $+/+$ & 1 & 8 \\
15 & M & 57 & Rt BG ICH & $-/-$ & 3 & 1 \\
16 & F & 64 & Schizophrenia, drug induced parkinsonism & $+/+$ & 3 & 8 \\
17 & F & 87 & Lt SDH & $+/+$ & 3 & 8 \\
18 & F & 77 & SAH, Lt precentral gyri ICH & $+/+$ & 8 & 8 \\
19 & F & 79 & SAH, ICH, SDH & $+/+$ & 23 & 8 \\
20 & M & 73 & Parkinson's disease & $+/+$ & 4 & 8 \\
$* 21$ & F & 56 & SAH, ICH d/t aneurysm rupture & $+/+$ & 4 & 8 \\
\hline
\end{tabular}

*Patients showing different VFSS depending on NGT presence. ICH, intracerebral hemorrhage; ChemoRTx, chemoradiation therapy; CVA, cerebrovascular event; SDH, subdural hemorrhage; SAH, subarachnoid hemorrhage

For interpretation of VFSS results, Rosenbek's penetration-aspiration scale (PAS) ${ }^{13}$ and diagnostic criteria for aspiration ${ }^{14}$ and videofluoroscopic dysphagia scale (VDS) ${ }^{15}$ were used. The diagnostic criteria for aspiration were as follows: normal, no entry of contrast material through the true vocal cords; mild, a trace of contrast materials through the true vocal cords; moderate, entry of $<10 \%$ of the bolus through the true vocal cords; severe, entry of $>10 \%$ of the bolus through the true vocal cords; and complete, frank aspiration of materials through the vocal cords without an observable reaction by the patient. VDS have been validated for assessing dysphagia in various disease including spinal cord injury, peripheral nervous system disorders an, and neurodegenerative diseases. ${ }^{16}$ VDS for VFSS with or without NGT were compared to investigate any functional difference in swallowing function.

The institutional review board of ethics has approved the study (IRB No. 2017-04-056-005). For statistical analysis, SPSS, $21^{\text {st }}$ edition (IBM,
USA) was used for descriptive analysis and paired $\mathrm{T}$ test comparing VDS scores with or without NGT.

\section{RESULTS}

The mean age of selected 21 patients was 73.2 years (SD 12.4 years, range $51-90$ years). The etiology of patients included stroke $(57.1 \%)$, Parkinson disease, neuromuscular junction disorder, malignancy, and others (Table 1). The mean PAS score was 6.8 (SD 2.3, range 1-8). Aspiration was observed in 18 patients, of whom 15 showed silent aspiration (76.2\%). These 15 patients scored 8 on PAS, indicating severe swallowing dysfunction, and included four patients whose VFSS results changed depending on NGT. In VFSS with NGT in situ, silent aspiration was observed in four patients, while VFSS without NGT revealed no aspiration. On the contrary, other 11 patients scoring 8 on PAS did not show any changes in VFSS results 
Table 2: Videofluoroscopic Dysphagia Scale with or without nasogastric tube

\begin{tabular}{llll}
\hline Diet type & Tube in situ & Tube removed & P value \\
\hline $\begin{array}{l}\text { Nectar } \\
\text { Pyriform sinus residue }\end{array}$ & $3.4 \pm 12.4$ & $3.2 \pm .4 .5$ & \\
Aspiration & $4.0 \pm 5.1$ & $2.6 \pm 4.9$ & 0.329 \\
$\quad$ Total & $32.6 \pm 16.1$ & $30.9 \pm 16.6$ & 0.056 \\
Pureed diet & & $0.033^{*}$ \\
$\quad$ Pyriform sinus residue & $3.4 \pm 4.5$ & $3.2 \pm .4 .5$ & \\
Aspiration & $2.9 \pm 4.8$ & $2.0 \pm 4.4$ & 0.329 \\
$\quad$ Total & $31.4 \pm 15.5$ & $30.4 \pm 15.8$ & 0.186 \\
\hline
\end{tabular}

Values are mean \pm standard deviation. ${ }^{*} p<0.05$ based on Paired T-test

depending on NGT in situ (Table 1). Comparing the results of VFSS with or without NGT for pureed diet and nectar drinking by paired $\mathrm{T}$ test revealed significant difference in total VDS score for nectar drinking (Table 2, $P<0.05$ ).

Patient 4 was a 51-year-old female with right hemiplegia due to ischemic infarction of the left posterior limb of internal capsule, basal ganglia, and thalamus. VFSS was performed 2 weeks after the onset. NGT in situ VFSS revealed supraglottic aspiration for both pureed diet and nectar, whereas VFSS without NGT did not show any aspiration.

Patient 12 was an 88-year-old female with a history of left thalamic intracerebral hemorrhage 13 years prior. She was admitted with aspiration pneumonia 3 months prior. She had been bedridden with dementia prior to admission. For both pureed diet and nectar drinking, subglottic aspiration was observed with NGT in situ, whereas no aspiration was observed in VFSS without NGT.

Patient 14 was an 84-year-old male with prior cerebrovascular events, 10 and 4 years ago. $\mathrm{He}$ was on non-modified oral diet despite intermittent aspiration symptoms. He was admitted for dyspnea owing to aspiration while eating 10 days prior to the VFSS. VFSS with NGT in situ showed supraglottic aspiration of nectar, while VFSS without NGT showed no aspiration.

Patient 21 was a 56-year-old female who was admitted with subarachnoid hemorrhage one month prior. Catheter insertion and coil embolization was performed for intracerebral hemorrhage owing to ruptured left middle cerebral artery bifurcation aneurysm. She was quadriplegic with severe weakness in the right limbs. VFSS with NGT in situ showed supraglottic aspiration for both pureed diet and nectar drinking, while VFSS without NGT showed no aspiration for either diet.

\section{DISCUSSION}

It is generally accepted to perform VFSS without NGT; however, patients showing different outcome from the expected suggests the presence of possible pitfalls in interpreting the results. Approximately 19\% of patients $(n=4)$ showed different VFSS results depending on NGT in situ . Such patients had a history of cerebrovascular events with variable duration of dysphagia and NGT feeding prior to VFSS from 1 week to 3 months. Moreover, they had a wide range of age from 51 to 90 years which suggests that the influence of NGT is not necessarily agedependent, although two patients were younger than the mean age. Neither the duration of NGT feeding nor the age of patients was associated with NGT dependent changes in VFSS. In addition, these four patients all scored PAS 8 and showed silent aspiration; however, the poor PAS score is not entirely related to NGT-dependent changes in VFSS results since 15 of 21 patients scored PAS 8. The specific reasons for changes in VFSS result in these patients are undetermined, however oral intake while NGT in situ may involve an additive risk of aspiration in some patients. Further, these results indicate that VFSS with NGT may entail significant information in deciding the diet method and mode of therapy for dysphagia.

Patient 4 showed subglottic aspiration for thin liquid and fluid drinking, and she may have been a suitable candidate for direct swallowing therapy, except for fluid. However, she was provided with indirect swallowing therapy with NGT in situ because VFSS results with NGT showed aspiration in pureed diet and nectar drinking. Similarly, patients 12, 14, and 21 underwent 4-8 weeks of indirect dysphagia therapy based on serially followed VFSS. Except for patient 12 who did not show adequate improvement to warrant oral 
diet with NGT removed, all three patients showed substantial recovery after indirect oral dysphagia therapy with NGT in situ. As of year, 2019, patients 4 and 14 are on chopped diet, while patient 21 is on a regular diet. Patients 4 and 14 are still on modified diet owing to persisting silent aspiration on fluid drinking. If VFSS with NGT had not been performed, the silent aspiration with NGT in situ would have gone unnoticed. Propitiously, they did not experience any pulmonary complications, including aspiration pneumonia during recovery.

Dysphagia is associated with various complications, including aspiration pneumonia, choking, increased mortality, and increased duration of hospital stays and rehabilitation. ${ }^{17}$ Recovery of patients without dysphagia-associated complications suggests VFSS with NGT in situ may help in identifying those with a high risk of aspiration during direct swallowing therapy, further enabling the safe transition from tube feeding to oral feeding.

The National Institute for Health and Care Excellence guideline of England recommends an initial 2-4-week trial of NGT feeding for patients in acute setting who are unable to swallow safely or obtain adequate energy and nutrients. ${ }^{18}$ Transition to oral feeding in patients receiving NGT feeding depends on the amount of adequate oral intake. ${ }^{19}$ Gradual increase in oral feeding while decreasing the corresponding amount fed via tube is introduced by Buchholz, who also recommends the discontinuation of tube feeding if $75 \%$ or more of nutrition requirements are satisfied by oral feeding for 3 days. ${ }^{20}$ It seems inevitable for some patients to experience swallowing with NGT in situ during the transition period. Therefore, the safety of actual swallowing as part of direct swallowing therapy with NGT in situ is an important concern for a successful swallowing therapy.

Early initiation of dysphagia therapy has been emphasized based on previous studies in which swallowing while NGT in situ did not worsen swallowing function. In studies utilizing fiberoptic endoscopic evaluation, NGT in situ did not worsen dysphagia in 25 acute stroke patients ${ }^{9}$ or 1260 inpatients with various etiologies. ${ }^{10}$ However, endoscopic evaluation has limited access to pharyngeal phase owing to the white-out period blocking the pharyngeal phase evaluation; therefore, it remains inconclusive whether oral diet therapy while NGT in situ changes the swallowing function.

Studies utilizing VFSS on aspiration risk of NGT showed similar results as in endoscopic evaluation: The results of VFSS with or without NGT for swallowing $1 \mathrm{~mL}$ fluid did not show any difference in stroke patients. ${ }^{21}$ However, fluid is usually not given orally in presence of NGT; moreover, the pureed diet or nectar thickening is usually applied as the initial material for swallowing therapy. Another study of 22 stroke patients swallowing $5 \mathrm{~mL}$ of thick and thin fluid did not show significant difference in VFSS results with or without NGT. Although above studies have concluded insignificant influence of NGT in swallowing, the safety of oral feeding while NGT in situ in all patients cannot be guaranteed as observed in this study.

Disruption in temporal coordination of swallowing physiology is associated with aspiration during VFSS..$^{22}$ Given the coordinated nature of swallowing process, the presence of NGT is likely to affect the swallowing physiology. Previously, a trend toward increased pharyngeal transit time with NGT in situ feeding was noted in some patients compared to swallowing without NGT. ${ }^{11}$ In a recent study of 30 stroke patients using NGT over two months showed a significant delay of pharyngeal transit time in NGT in situ VFSS. ${ }^{23}$ The delay in pharyngeal transit time while NGT in situ may suggest possible influence of NGT on reflexive sensory-motor response.

Swallowing involves the activation of multiple sensory fibers over multiple parallel pathways ${ }^{24}$. The sensory stimuli triggering and modulating swallowing include tactile, chemical, thermal, and combined stimulus modalities. ${ }^{25}$ Stimuli such as smoking is reported to alter the thresholds for laryngeal reflexes and pharyngeal swallowing. Similarly, the NGT may have acted as a stimulus altering the swallowing reflexes by contacting the junction of naso- and oropharynx which has the highest density of pharyngeal sensory receptors. ${ }^{26}$

Another potential factor contributing to the change observed in VFSS with NGT is laryngeal sensitivity. In a cross-sectional study of stroke patients, laryngeal sensitivity showed negative correlation with laryngeal penetration and tracheal aspiration. ${ }^{27}$ Patients showing varying results may have been reflecting poor laryngeal sensitivity associated with NGT placement. The influence of NGT during VFSS could vary especially in patients with good integrity of reflexive sensorymotor responses; however, most patients in this study had severe dysphagia unable to show effective clearance of the penetrance. Although further study is needed to confirm the relation, patients showing change with NGT in situ need to be addressed cautiously when applying direct 
swallowing therapy. Performing VFSS with NGT in situ for pureed diet and nectar drinking is feasible and time efficient as it can be addressed prior to conventional VFSS before removing NGT. Although specific guidelines for patients in need of NGT in situ VFSS are yet to be provided, VFSS with NGT is a safe parameter to distinguish those unsuitable for direct swallowing training.

As weaning from tube feeding to oral intake is patient dependent ${ }^{28}$, individual traits that are prone to affect swallowing function despite NGT should be taken into consideration. Cognitive dysfunction is a known factor attributing to delay of NGT weaning. Some patients in this study had a long duration of illness prior to VFSS, including dementia. Loss of bolus control and reduced lingual control are reported as the most common features of swallowing disorders in patients with severe brain injury. ${ }^{29}$ Delay in the oral phase was frequently observed in these patients. The poor cognitive function in most patients could have been associated with poor swallowing function.

This study has some limitations. First, the retrospective nature and small number of patients limit the generalization of our results. In addition, the variation in study population, including the etiology, duration of NGT feeding, and short time interval between NGT in situ and removal for VFSS all need further consideration. Another limitation is the lack of information regarding other risk factors for dysphagia in some patients, including the level of cognitive function, concomitant dysarthria, aphasia, and level of consciousness, which may also have contributed to VFSS results.

Nevertheless, to the best of our knowledge, this is the first study to document the need of NGT in situ VFSS for dysphagia therapy. For nectar drinking, the VDS scores were significantly greater in NGT in situ which indicates great diet limitation and more severe dysphagia with NGT. This NGT induced aggravation in swallowing were observed regardless of NGT duration that patients had variable NGT duration from 1 week to 12 weeks. Although, the cut-off value indicative of NGT in situ cannot be concluded from these results, the NGT in situ VFSS seems indicative for screening candidates of direct swallowing therapy at least for nectar drinking regardless of NGT duration.

Further study consisting of a larger study population with variable causes of dysphagia would be necessary. Moreover, the specificity and sensitivity of NGT in situ VFSS must be studied to provide better insights into the tolerance and safety of swallowing therapy with NGT in situ in patients with dysphagia.

In conclusion, in our study, VFSS results differed in 19\% of patients with NGT in situ. All patients had dysphagia due to stroke, showed a PAS score of 8 with silent aspiration. Based on VFSS with NGT, the mode of therapy for dysphagia was determined, through which dysphagia in these patients improved without associated pulmonary complications. These results suggest that VFSS with NGT in situ should be considered in patients with dysphagia in conjunction with conventional VFSS, especially in patients with high PAS scores with silent aspiration, to obtain additional information regarding the risks associated with direct dysphagia therapy.

\section{DISCLOSURE}

\section{Conflict of interest: None}

Financial support: This study is supported by a research grant of Research Institute of Rehabilitation Medicine, Yonsei University for 2020.

\section{REFERENCES}

1. Hoffmann S, Malzahn U, Harms H, et al. Development of a clinical score (A2DS2) to predict pneumonia in acute ischemic stroke. Stroke 2012;43(10):2617-23.

2. Morgan AS, Mackay LE. Causes and complications associated with swallowing disorders in traumatic brain injury. J Head Trauma Rehabil 1999;14(5):45461.

3. Muller J, Wenning GK, Verny M, et al. Progression of dysarthria and dysphagia in postmortem-confirmed parkinsonian disorders. Arch Neurol 2001;58(2):25964.

4. Mulcahy KP, Langdon PC, Mastaglia F. Dysphagia in inflammatory myopathy: self-report, incidence, and prevalence. Dysphagia 2012;27(1):64-9.

5. Kuhnlein P, Gdynia HJ, Sperfeld AD, et al. Diagnosis and treatment of bulbar symptoms in amyotrophic lateral sclerosis. Nat Clin Pract Neurol 2008;4(7):366-74

6. Wirth R, Dziewas R, Beck AM, et al. Oropharyngeal dysphagia in older persons - from pathophysiology to adequate intervention: a review and summary of an international expert meeting. Clin Interv Aging 2016;11:189-208.

7. Crary MA, Groher ME. Reinstituting oral feeding in tube-fed adult patients with dysphagia. Nutr Clin Pract 2006;21(6):576-86.

8. Carnaby G, Hankey GJ, Pizzi J. Behavioural intervention for dysphagia in acute stroke: a randomised controlled trial. Lancet Neurol 2006;5(1):31-7.

9. Dziewas R, Warnecke T, Hamacher C, et al. Do nasogastric tubes worsen dysphagia in patients with acute stroke? BMC Neurol 2008;8(1):28. 
10. Leder SB, Suiter DM. Effect of nasogastric tubes on incidence of aspiration. Arch Phys Med Rehabil 2008;89(4):648-51.

11. Wang TG, Wu MC, Chang YC, Hsiao TY, Lien IN. The effect of nasogastric tubes on swallowing function in persons with dysphagia following stroke. Arch Phys Med Rehabil 2006;87(9):1270-3.

12. Logemann JA. Manual for the videofluorographic study of swallowing. Vol 2: Pro-ed Austin; 1993.

13. Rosenbek JC, Robbins JA, Roecker EB, Coyle JL, Wood JL. A penetration-aspiration scale. Dysphagia 1996;11(2):93-8.

14. Mann G, Hankey G, Cameron D. Swallowing function after stroke: prognosis and prognostic factors at 6 months. Stroke 1999;30(4):744.

15. Han TR, Paik NJ, Park JW, Kwon BS. The prediction of persistent dysphagia beyond six months after stroke. Dysphagia 2008;23(1):59-64.

16. Kim J, Oh BM, Kim JY, Lee GJ, Lee SA, Han TR. Validation of the videofluoroscopic dysphagia scale in various etiologies. Dysphagia 2014;29(4):438-43.

17. Sura L, Madhavan A, Carnaby G, Crary MA Dysphagia in the elderly: management and nutritional considerations. Clin Interv Aging 2012;7:287-98.

18. Care NCCfA. Nutrition support for adults: oral nutrition support, enteral tube feeding and parenteral nutrition. 2006.

19. Crary MA, Groher ME. Reinstituting oral feeding in tube-fed adult patients with dysphagia. Nutr Clin Pract 2006;21(6):576-86.

20. Buchholz AC. Weaning patients with dysphagia from tube feeding to oral nutrition: A proposed algorithm. Can J Diet Pract Res 1998;59(4):208-14.

21. Kim G, Baek S, Park HW, Kang EK, Lee G. Effect of nasogastric tube on aspiration risk: Results from 147 patients with dysphagia and literature review. Dysphagia 2018:1-8.

22. Martin-Harris B, Brodsky MB, Michel Y, Ford CL, Walters B, Heffner J. Breathing and swallowing dynamics across the adult lifespan. Arch Otolaryngol Head Neck Surg 2005;131(9):762-770.

23. Wang ZY, Chen JM, Ni GX. Effect of an indwelling nasogastric tube on swallowing function in elderly post-stroke dysphagia patients with long-term nasal feeding. BMC Neurol 2019;19(1):83.

24. Capra NF. Mechanisms of oral sensation. Dysphagia 1995;10(4):235-47.

25. Steele CM, Miller AJ. Sensory input pathways and mechanisms in swallowing: a review. Dysphagia 2010;25(4):323-33.

26. Bradley RM, Mistretta CM. Fetal sensory receptors. Physiol Rev 1975;55(3):352-82.

27. Onofri SM, Cola PC, Berti LC, da Silva RG, Dantas RO. Correlation between laryngeal sensitivity and penetration/aspiration after stroke. Dysphagia 2014;29(2):256-61.

28. Boullata JI, Carrera AL, Harvey L, et al. ASPEN safe practices for enteral nutrition therapy JPEN $J$ Parenter Enteral Nutr 2017;41(1):15-103.

29. Mackay LE, Morgan AS, Bernstein BA. Swallowing disorders in severe brain injury: risk factors affecting return to oral intake. Arch Phys Med Rehabil 1999;80(4):365-71. 